\title{
Perspective Actionelle Visa-a-Vis L'enseignement du Français Langue Entrangele au Nigeria : Problem et Vision
}

\author{
Scholastica Ezeodili \\ *http://dx.doi.org/10.4314/ujah.v15i2.7
}

\section{Résumé}

La langue française s'inscrit comme l'une des langues étrangères les plus enseignées au cadre universitaire nigérian. Ayant fait entrée au pays il y a plusieurs décennies, un progrès rapide se marque dans l'enseignement et apprentissage de cette langue étrangère. Toutefois, il ne va pas sans problèmes aussi bien au niveau de manque des matériaux pédagogiques, ainsi que la méthodologie d'enseignement qui ne cesse d'évoluer. À l'heure actuelle, c'est la perspective actionnelle qui se pose comme la méthodologie la plus récente. C'est une nouvelle perspective d'enseignement et d'apprentissage des langues considérant les apprenants comme des acteurs sociaux ayant à accomplir des tâches langagières et non-langagières. Dans cette communication, nous avons comme objectif de discuter cette nouvelle perspective en fonction de son adaptation dans l'université nigériane.

\section{Abstract}

French language is positioned as one of the most taught foreign languages in the Nigerian university. Having penetrated into the country some decades ago, an unprecedented progress has been recorded in its teaching and learning. However, it has not been without some 


\section{L'enseignement du Français Langue Entrangele au Nigeria : Problem et Vision}

challenges ranging from inadequate and insufficient pedagogical materials to the continual change in the teaching methods. Currently, the Common European Framework of Reference for European languages is based on the new approach 'perspecitve actionnelle' an offshoot of the communicative approach which came into limelight in the 80s.. This method considers the learner as a social actor having to accomplish tasks that are both linguistic and non linguistic tasks in the society. Meanwhile, its application is not yet popular in Nigeria. In this communication therefore, the new method has been discussed with a view to finding out its application implication in Nigerian universities.

\section{Introduction}

La langue française occupe actuellement une place enviable dans le monde politique, culturelle, scientifique et aussi technologique. Le Nigeria, bien qu'il soit un pays anglophone, ne cesse de s'adapter continuellement aux défis qu'impose l'enseignement de cette langue vivante dans nos institutions. Par conséquent, les différentes méthodologies qui sont tantôt favorables et adaptables tantôt défavorables et inadéquats continuent à se mettre en œuvre compte tenue que c'est la langue étrangère la plus enseignée et apprise dans les cadres secondaires et universitaires.

Aujourd'hui, plus de $91 \%$ des élèves étudient le français dans le premier cycle du secondaire et le français est considéré comme la plus importante langue étrangère parlée au Nigeria. (Le français au Nigeria Université Laval. http: www.tlfq.ulaval.ca/ax//afrique/nigeria.htm avril 2010).

Depuis quelques décennies, la méthodologie communicative qui est supposée se pratiquer remplace les 
anciennes méthodologies. Néanmoins, l'adaptabilité ne cesse d'être mythique dans plusieurs institutions considérant ses exigences. Laditan, lors de la 16eme édition de la francophonie au Nigeria organisé dans les 10 Alliances françaises a rappelé la nécessité d'abandonner la méthode traditionnelle et d'enseigner le français comme langue vivante. Considérant le but à atteindre, plusieurs méthodologies d'enseignement sont déjà mise en place depuis, afin de faciliter l'apprentissage et pour s'adapter au besoin culturel et technologique du moment. À l'heure actuelle l'approche communicative adoptée par les pédagogues et les didacticiens de langue reste toujours au niveau expérimental.

Aujourd'hui, c'est la perspective actionnelle qui est proposée par le Cadre Européen Commun de référence (CECR) avec ses complexités d'application. Selon Cuq,

Le CECR est un document de référence élaboré à l'initiative et au sein du conseil de l'Europe, (...) et destiné, d'une part, à faciliter les échanges entre différents acteurs du domaine de l'enseignement et de l'apprentissage des langues et, d'autre part, à assister chacun de ces différents acteurs dans la réflexion et la prise de décision intéressante son propre domaine d' intervention (38).

Cette présente étude mettra en lumière quelques points clés de la nouvelle approche. Pour y parvenir, un bref rappel historique de quelques anciennes méthodologies sera chronologiquement exploité d'abord suivi par un exposé des grandes lignes de la nouvelle approche. Ensuite, nous allons déterminer la possibilité de son adaptation à l'université au Nigeria.

\subsection{Les Principaux Courants Methodologiques}

\subsection{Méthodologie Traditionnelle}


C'est la méthodologie qui est basée sur l'enseignement des règles grammaticales, la traduction thème-version et la connaissance des grands textes littéraires. La langue est conçue comme un ensemble des règles et des exceptions observables dans les textes. Parler la langue, c'est traduire, donc la traduction est l'activité de la classe avec les activités langagières de compréhension écrite et expression écrite. La grammaire est enseignée d'une manière déductive. L'enseignant dispose l'instruction tout en proposant des phrases qui sont souvent artificielles.

\subsection{La Méthodologie Directe /Active}

Celle-ci constitue une approche naturelle de l'apprentissage d'une langue étrangère fondée sur l'observation de l'acquisition de la langue maternelle par l'enfant. L'apprenant pense directement en langue étrangère tout en évitant le recours à la langue maternelle, l'objectif étant de faire parler l'apprenant en langue étrangère sans passer par la langue maternelle.

\subsection{Méthodologie Audio-Orale}

Le but est en principe de parvenir à communiquer en langue étrangère .On accorde la priorité à l'oral. La langue est considérée comme un ensemble d'habitudes, et d'automatisme linguistiques et donc résultant à l'utilisation spontanée des formes linguistiques. La grammaire dans cette méthodologie est considérée comme une liste de structures.

\subsection{L'Approche Communicative}

L'approche communicative se donne pour objectif de faire acquérir à l'élève la langue dans la variété de ses registres et usages en tentant de ne pas séparer langue et civilisation (Berard 16). Elle s'est développée dans l'enseignement du 
FLE à partir de 1975 dans un mouvement par rapport aux méthodes précédentes. L'objectif de cette méthodologie est d'aider l'apprenant à communiquer dans la langue étrangère. L'emphase n'est pas seulement sur la compétence linguistique mais aussi la compétence socioculturelle.

D'après Germain, cette approche surgit avec la création du Niveau-seuil en 1976 pour le cas du français (qui suit le modèle du Threshold Level English). http://www.dissertationsgratuites.com/dissertations/La-

Perspective-Actionnelle-Evolution-De-

1\%E2\%80\%99Approche/103492.html

La langue est conçue comme un instrument de communication et d'interaction sociale. Il ne suffirait pas de connaitre les règles grammaticales de la langue étrangère pour communiquer, il faut en plus connaitre les règles d'emploi de cette langue. L'emphase est plutôt sur les tâches à accomplir au lieu des difficultés linguistiques.

Voici sur forme schématique, l'histoire des méthodologies adapté de O. GLAIN - LGT A.Thomas, Roanne :

\begin{tabular}{|c|c|c|c|c|c|}
\hline & $\begin{array}{l}\text { Méthodol } \\
\text { ogie } \\
\text { traditionn } \\
\text { elle } \\
\text { 19ème } \\
\text { siècle }\end{array}$ & $\begin{array}{l}\text { Méthodol } \\
\text { ogie } \\
\text { active / } \\
\text { directe } \\
1900- \\
1960\end{array}$ & $\begin{array}{l}\text { Méthodol } \\
\text { ogie } \\
\text { audiovisu } \\
\text { elle } \\
1960- \\
1980\end{array}$ & $\begin{array}{l}\text { Approche } \\
\text { Communic } \\
\text { ative } \\
1980-1990\end{array}$ & $\begin{array}{l}\text { Approche } \\
\text { actionnelle } \\
2000\end{array}$ \\
\hline $\begin{array}{l}\text { Parler } \\
\text { une } \\
\text { langue } \\
\text { c'est... }\end{array}$ & traduire. & $\begin{array}{l}\text { penser et } \\
\text { Parler } \\
\text { 'directem } \\
\text { ent' }\end{array}$ & $\begin{array}{l}\text { réagir à } \\
\text { des } \\
\text { situations } \\
\text {. }\end{array}$ & $\begin{array}{l}\text { échanger, } \\
\text { informer, } \\
\text { s'informer }\end{array}$ & $\begin{array}{l}\text { Agir } \\
\text { Ensemble }\end{array}$ \\
\hline $\begin{array}{l}\text { Activit } \\
\text { és }\end{array}$ & $\begin{array}{l}\text { Traductio } \\
\mathrm{n}\end{array}$ & Exercices & Exercices & $\begin{array}{l}\text { Simulation } \\
\text { et }\end{array}$ & \\
\hline
\end{tabular}




\begin{tabular}{|c|c|c|c|c|c|}
\hline $\begin{array}{l}\text { de } \\
\text { classe }\end{array}$ & & $\begin{array}{l}\text { de } \\
\text { transform } \\
\text { ation } \\
\text { 'substituti } \\
\text { on, } \\
\text { questions } \\
\text { / } \\
\text { réponses. }\end{array}$ & $\begin{array}{l}\text { visant les } \\
\text { automatis } \\
\text { mes. } \\
\text { Exercices } \\
\text { structurau } \\
\text { x. }\end{array}$ & $\begin{array}{ll}\text { jeux } & \text { de } \\
\text { rôles. } & \end{array}$ & \\
\hline $\begin{array}{l}\text { Activit } \\
\text { és } \\
\text { langagi } \\
\text { ères }\end{array}$ & $\mathrm{CE}(\mathrm{EE})$ & $\begin{array}{l}\text { EO } \\
\text { Combinai } \\
\text { son } \\
\text { CE-EO }\end{array}$ & $\begin{array}{l}\text { Combinai } \\
\text { son } \\
\text { CO-EO }\end{array}$ & $\begin{array}{l}\text { Juxtapositi } \\
\text { on } \\
\text { CO-EO- } \\
\text { CE EE }\end{array}$ & $\begin{array}{l}\text { Articulatio } \\
\mathrm{n} \\
\text { CO-EO-CE } \\
\text { EE }\end{array}$ \\
\hline $\begin{array}{l}\text { Méthod } \\
\text { es } \\
\ldots\end{array}$ & $\begin{array}{l}\text { Indirecte, } \\
\text { réflexive, } \\
\text { applicativ } \\
\mathrm{e}\end{array}$ & $\begin{array}{l}\text { Directe, } \\
\text { active }\end{array}$ & $\begin{array}{l}\text { Directe, } \\
\text { active, } \\
\text { répétitive }\end{array}$ & $\begin{array}{l}\text { Directe, } \\
\text { active, } \\
\text { ) Réflexive }\end{array}$ & $\begin{array}{l}\text { Directe } \\
\text { active } \\
\text { réflexive }\end{array}$ \\
\hline $\begin{array}{l}\text { Pilotag } \\
\text { e } \\
\text { par... }\end{array}$ & $\begin{array}{l}\text { Les } \\
\text { exemples } \\
\text { phrases } \\
\text { isolées }\end{array}$ & \multicolumn{3}{|l|}{$\begin{array}{l}\text { Les } \\
\text { document } \\
\text { s }\end{array}$} & $\begin{array}{l}\mathrm{La} \text { tache } \\
\text { (scolaire/so } \\
\text { ciale) }\end{array}$ \\
\hline
\end{tabular}

\subsection{Les Grandes Lignes de la Perspective Actionnelle}

\section{1 La Perspective actionnelle et le Cecrl}

La perspective actionnelle est une des quatre évolutions proposées par le Cadre Européen Commun de Référence pour les langues (CECRL) rédigé par le conseil de l'Europe en 2012. Postérieurement, à la fin des années 1990, la perspective actionnelle fait son apparition à la suite de la création du Cadre Commun de Référence pour les Langues.

Le cadre qui n'est ni un manuel ni un référentiel de langue est un outil fournissant une base commune pour l'enseignement et l'apprentissage des langues ayant comme les 4 axes de réflexions les suivants : 
Les six niveaux communs de référence- AI, A2, B1, $\mathrm{B} 2, \mathrm{C} 1, \mathrm{C} 2$

Les cinq activités langagières (écouter, lire, prendre part à une conversation, s'exprimer oralement en continu, et écrire)

Les 4 composantes de la compétence communicative

Une nouvelle perspective de l'enseignement qui est la perspective actionnelle

(Paola Bagnoli et al www. Ph-ludwigsburg.de)

Notre emphase se mettra particulièrement sur le quatrièmePerspective Actionnelle. C'est une nouvelle perspective d'enseignement et d'apprentissage des langues qui considère avant tout l'usager de la langue comme des acteurs sociaux ayant à accomplir des tâches (qui ne sont pas seulement langagières) dans les circonstances et un environnement donnés à l'intérieur d'un domaine d'action particulier (Tagliante Christiane 36). L'apprenant est amené à agir avec les autres en contexte sociale aussi pas seulement en contexte scolaire. Dans les mots de Puren Christian 'Le nouvel enjeu n'est pas seulement de communiquer mais d'agir' (37). Selon Bérard, les activités s'accomplissent en groupe. Les principes fondamentaux et pédagogiques de l'approche actionnelle dans le CECR incluent la tâche, le contexte, les activités langagières, impliquant l'exercice de la compétence à communiquer langagièrement, dans un domaine déterminé, pour traiter un ou des textes en vue de réaliser une tâche, la stratégie, les actes de parole, la compétence (linguistique, sociolinguistique et pragmatique), objectif. C'est autour de la tâche que se déroulent les autres activités.

Le principal objectif ciblé dans cette formation est donc de réaliser des actions communes, collectives en langue étrangère, agir avec l'autre. Il a aussi comme objectif de 
produire les apprenants avec l'apprentissage transcendant le clivage des différentes disciplines.

\subsection{Notion De Tache}

Une tâche est une activité qui présente un problème à résoudre, un manque qui crée le besoin et oblige à agir. (http:///www.aplv-langues modernes.org).

D’après le CECR :

Les tâches ou activités sont l'un des faits courants de la vie quotidienne dans les domaines personnels, public, éducationnel et professionnel. L'exécution d'une tâche par un individu suppose la mise en œuvre stratégique de compétences données, afin de mener à bien un ensemble d'actions finalisées dans un certain domaine avec un but défini et un produit particulier (121).

Donc, la tâche est orientée vers un but à atteindre, un problème à résoudre. Selon Bérard, 'une tache permet de centrer l'attention de l'apprenant sur ce qu'il doit réaliser et sur les compétences à mettre en œuvre pour le faire'(7).

Medioni a résumé le terme de la tâche en six points dont nous tirerons une de ses définitions :

Une tâche est une activité interactive qui met en relation des interlocuteurs ayant des choses à se dire et des actions à produire ensemble; elles impliquent à la fois la coopération et la confrontation susceptible de relancer le questionnement quand on le croit épuisé par les contradictions qu'elle risque de faire surgir.

(http:///www.aplv- langues modernes.org)

C'est une activité qui présente un problème à résoudre, un manque qui crée le besoin et oblige à agir. La tache est "authentique" en ce qu'elle consiste en une activité quotidienne, dans le cadre du travail, des études ou de la vie privée (Conseil de l'Europe, 2001 : 121). 
Dans l'accomplissement des tâches, l'apprenant doit faire appel à des compétences langagières, mais aussi à ses habiletés "cognitives, affectives, volitives et l'ensemble des capacités que possède et met en œuvre l'acteur social" (Conseil de l'Europe, 2001 : 15).

\subsection{L'approche Communicative Et La Perspective Actionnelle}

Depuis les années 1980, c'est l'approche communicative qui est soutenue pour l'enseignement de la langue vivante. Quelques pédagogues conçoivent que la perspective actionnelle est une évolution de cette dernière. En ce sens, Robert et Rosen synthétisent les évolutions marquant le tournant de l'approche communicative à la perspective actionnelle. D'après eux, les objectifs ciblés par la formation dans la méthodologie précédente est celui d'apprendre à communiquer en langue étrangère et de parler avec l'autre. De sa part, la présente approche vise à réaliser les actions communes, collectives, en langue étrangère et donc agir avec l'autre. Quoiqu' il existe plusieurs points de rencontre, des écarts s'y soulignent aussi surtout au niveau de leurs principes forts et objectifs ciblés, l'évaluation et les activités privilégiées.

Dans l'approche communicative, on apprend à communiquer en langue étrangère tout en parlant avec l'autre. La perspective actionnelle, de sa part, souligne la réalisation des actions communes et collectives en langue étrangère tout en agissant avec l'autre. En plus, la production /réception de l'oral et de l'écrit : les jeux de rôle et de simulation constituent des activités privilégiées liées à l'approche communicative. En revanche, l'interaction/médiation caractérisé par la pédagogie par taches et par projet forment les activités langagières de la perspective actionnelle. D'ailleurs, l'évaluation dans l'approche communicative est basée sur la 
compétence de la communication. Cependant, les compétences langagières et des compétences générales individuelles et sociales constituent l'évaluation en perspective actionnelle.

En plus, la centration est sur l'apprenant qui est sujet et acteur principal de l'apprentissage et non objet ou destinataire d'une méthode. La dernière, de sa part, accorde l'importance à la co-construction du sens (accent mis sur l'agir communicationnelle). Ici, la centration est sur le groupe (classe), la dimension collective, impliquant que l'apprenant devient un citoyen actif et solidaire. Bref, la perspective actionnelle reflète le passage du paradigme de la communication au paradigme de l'action.

\subsection{Le Traitement De La Perspective Actionnelle Dans L'enseignement Du Français A L'universite}

Peut-on s'en tirer de la perspective actionnelle comme méthodologie d'enseignement des différents domaines de la langue - grammaire, civilisation, linguistique, phonétique et littérature au Nigeria?

Evidemment, l'approche facilite la compétence générale dans la langue étrangère. Comme son nom indique, elle se base incontestablement sur l'action. Avec la perspective actionnelle, les apprenants acquièrent rapidement la compétence à la fois linguistique, sociolinguistique et pragmatique en langue étrangère. Compte tenu du niveau des apprenants, l'enseignant s'insère au cadre du médiateur. La tâche, une fois bien définie, les apprenants d'une façon collective s'orientent à agir.

Très essentiel est l'Internet, fournisseur d'un grand avantage car plusieurs activités s'y proposent dans les différents domaines et selon le niveau de l'apprenant. 
En grammaire par exemple, plusieurs sites sont proposés où les apprenants vont eux-mêmes accéder.

\subsection{Le Tbi- Tableau Blanc Interactif}

Le tableau blanc interactif (TBI), ou tableau numérique interactif (TNI), nommé aussi parfois tableau pédagogique interactif (TPI), est un système de vidéo projection numérique qui vise à remplacer le tableau avec craie ou crayon effaçable en lui ajoutant parfois des haut-parleurs. www.educatechmaroc.com/?p=423

Il existe aussi un dispositif mobile interactif (DMI), facilement transportable.

Un TPI fonctionne selon deux façons : le stylet (ou le doigt dans certains modèles) est reconnu par un système de capture de mouvement grâce à une caméra ou à un dispositif placé au niveau de l'écran tactile. Le rendu visuel se fait par vidéo projecteur sur un écran blanc qui supporte souvent les marqueurs effaçables à sec et qui sont actuellement de plus grande taille que les systèmes de captation internes (écrans tactiles) ou externes (fixés ou ajoutés à l'écran). Le tableau est

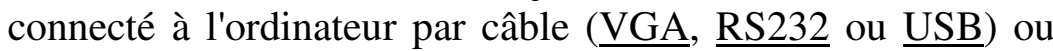
sans (Wifi, Bluetooth). Fr.m.wikipedia.org Tableau blanc interactif

Avec certains modèles, le professeur et l'élève peuvent avoir chacun respectivement une tablette graphique connectée en wifi et un stylet pour charger les écrans et écrire sur le tableau. Il est utilisé dans le cadre de la mise en place des TIC et des TICE. fr.wikipedia.org/wiki/Tableau-blanc-interactif Un écran blanc tactile est relié à un ordinateur via un câble (généralement USB) ou sans fil. Un vidéoprojecteur se charge d'afficher l'écran de l'ordinateur, sur le tableau blanc. Il est possible d'effectuer à la main ou à l'aide d'un stylet (parfois les deux selon les modèles), tout ce qui est possible de réaliser à l'aide d'une souris, sur un format d'écran assez important 
(jusqu'à plus de $2 \mathrm{~m}$ de diagonale). Le tableau est généralement fourni avec un logiciel dédié, qui permet de dessiner dessus d'une façon adaptée à l'écran tactile. Avec une telle technologie,

\subsection{La Mise En Pratique}

La nouvelle approche pourrait s'insérer dans le cadre universitaire à partir des matériels disponibles du TBI, vidéoprojecteur, les $\mathrm{CD}$ rom et les autres. Nous tenons à esquisser, d'après notre expérience, quelques unes des difficultés face à l'adaptation de la perspective actionnelle dans une classe de langue à l'université nigériane. Bien que cette perspective soit un grand succès en Europe et un changement opportun, considérant son adaptabilité au milieu universitaire nigérian reste contestée étant donné des facteurs qui s'imposent.

En premier lieu, c'est une perspective fondée sur les six niveaux proposés par le CECRL. Les étapes à suivre pour pouvoir arriver à une tâche finale sont très rigoureuses et complexes et par conséquent peuvent ne pas bien rentrer dans le cadre du programme compte tenue des exigences du programme scolaire, En plus, considérant le programme universitaire et l'effectif de la classe, la participation de tous les groupes d'apprenants dans le temps imparti posera aussi des problèmes.

En principe, c'est une démarche qui exige beaucoup d'activités et d'actions donc demande beaucoup de temps. Par conséquent, la possibilité d'achever le contenu du cours trimestriel dans les filières surtout de la linguistique, la grammaire, et la littérature reste un peu redoutable. Il existe en plus le problème d'espace pour faire bouger librement les apprenants et l'enseignant. C'est une approche qui demande le développement systématique des cinq compétences 
langagières or ces compétences manque chez quelques enseignants. En plus, un grand nombre de nos enseignants ne sont pas à jour avec la nouvelle technologie du TBI (tableau blanc interactif), vidéoprojecteur et d'autres outils multimédia. Ces appareils avec leurs prix exorbitants sont difficiles à procurer. Le tableau blanc interactif (TBI), ou tableau numérique interactif (TNI), nommé aussi parfois tableau pédagogique interactif (TPI), est un système de vidéo projection numérique qui vise à remplacer le tableau avec craie ou crayon effaçable en lui ajoutant parfois des hautparleurs. Il existe aussi un dispositif mobile interactif (DMI), facilement transportable.

L'enseignement du français au Nigeria est très scolaire et on emploie des méthodes démodées. L'approche actionnelle, de sa part, n'est donc pas une approche facile à mettre en place lorsqu'on n'est pas suffisamment formé, tant au niveau linguistique que pédagogique.

D'après la discussion menée jusqu'ici l'environnement de l'enseignement/apprentissage en grand mesure détermine l'adaptabilité et praticabilité d'une méthode donnée. Etant donné l'objectif général et varié de l'apprentissage du français au Nigeria, sa potentialité reste un mythe. On ne l'apprend pas forcement pour agir mais pour communiquer.

\section{Conclusion}

La nouvelle approche de l'enseignement des langues vivantes est certainement assez louable considérant la situation actuelle d'immigration et d'émigration. Dans ce cas, l'apprenant se tourne aussi vers la société où il doit incontestablement agir. Bien que cette approche soit pédagogiquement rigoureuse, elle présente une ambiance passionnante et stimulante pour les apprenants et l'enseignant. Le dernier sera en mesure de bien planifier ces cours de façon à relever les taches. Les apprenants, acteurs principaux de l'acte de l'acquisition et de 
l'apprentissage aussi bien que les enseignants doivent s'apprêter à prendre des initiatives à l'exécution de leurs propres activités d'apprentissage.

Puisqu' un grand nombre d'enseignants ne sont pas encore à jour avec la nouvelle approche, nous posons en principe qu'une formation continu des enseignants soit mise en place. En plus, on suggère également la coopération accentuée des gouvernements nigérians/ français pour la provision des matériels modernes comme le TBI, et les projecteurs pour la stimulation d'action chez les apprenants. Souvenons-nous aussi qu'aucune méthodologie n'est parfaite et donc la meilleure pour nous est la méthode éclectique.

\author{
Scholastica Ezeodili \\ Department of Modern \\ European Language \\ Nnamdi Azikiwe University \\ scholasticaezeodili@yahoo.co.uk
}




\section{Euvres Citées}

Ana Rodriguez Seara. 'L'évolution des méthodologies dans l'enseignement du français langue étrangère depuis la méthodologie traditionnelle jusqu'a nos jours' http://www.uned.es/ca-tudela/revista/n001/art-8.htm mise en ligne le 26 janvier, 2004 consulté en 2013.

Aurélie, Bernard. 'Perspective Actionnelle et TICE' posté le 29/4/2010

Berard, E. L'approche communicative: Théorie et pratique. Paris: Clé International, 1991.

Conseil de l'Europe. Cadre Européen Commun de Référence pour les langues. Paris : Les éditions Didier, 2001.

Cuq, J-P . Dictionnaire de didactique du français langue étrangère et seconde. Paris : Cle Int/ SEJER, 2003.

Elke Nissen, «Variations autour de la tâche dans l'enseignement / apprentissage des langues

aujourd'hui », Alsic [En ligne], Vol. 14|2011, mis en ligne le 11 octobre 2011, Consulté le 04 octobre 2012. URL :http://alsic.revues.org/2344 ; DOI : 10.4000/alsic. 2344

La Perspective Actionnelle: Evolution De L'approche Communicatif?

DissertationsGratuites.com. Récupérée 11, 2010, à partir de http://www.dissertationsgratuites.com/dissertations/La -Perspective-Actionnelle

Evolution-De-1\%E2\%80\%99Approche/103492.html

La perspective actionnelle: Didactique et pédagogie par l'action en Interlangue.

Trabajo presentado en el 3er. Foro de Lenguas de ANEP, 8 10 de octubre 2010, Montevideo www.eduscol.com .

Le français au Nigeria .Université Laval. http : www.tlfq.ulaval.ca/ax//afrique/nigeria.htm

Dernière mise à jour28 octobre, 2010. 
'Le Tableau blanc interactif'. www.educatechmaroc.com/?p=423 mise à jour le 29 mars 2010 , consulté en octobre, 2013.

Medioni Marie-Alice 'L'enseignement -apprentissage des langues : un agir ensemble qui s'affirme.' Association des professeurs de langues vivantes. (http:///www.aplvlangues modernes.org)

Nurten Özçelik. 'L'enseignement et l'apprentissage de la grammaire en classe du FLE' http : //egitimdergi.omu.edu.tr/DergiPdfDetay.asp $\times \% 3 F I D \%$ 3D100 (2012) 31,1,175- 186.

O. Glain - LGT A Thomas, Roanne 'L'approche actionnelle' appd.anglais.free.fr/IMG/pdf/l'approche actionnelle.pdf mardi 24 octobre 2006, Lyon.

Paolo Bagnoli, Edward Don, Rosina Praderi et Veronique Ruel . 'La perspective actionnelle :Didactique et pedagogique par action en Interlangue 'mise en ligne en 2010 www.ph-ludwigsburg.de

Puren, Christian, Bertocchini, Paola et Costanzo Edvige . Se former en Didactique des langues . Paris : 1998.

Rivenc, Paul. 'Et la grammaire dans tout cela ?' Revue de phonétique appliquée. s: 61.s. 339- 351, Mons Belgique : Université de l'Etat.

Robert Jean- Pierre et Rosen Evelyn. Dictionnaire Pratique $d u$ CECR. Paris : Ophrys, 2002.

Tagliante, Christine. L'Evaluation et le cadre européen commun. Paris : Clé International, 2005.

(http:///www.aplv-langues modernes.org).

Fr.m.wikipedia.org Tableau blanc interactif mise à jour en 2007 